\title{
THE RELATIONSHIP OF METEORITIC PARENT BODY THERMAL HISTORIES AND ELECTROMAGNETIC HEATING BY A PRE-MAIN SEQUENCE T TAURI SUN
}

\author{
C. P. SONETT \\ NASA Ames Research Center
}

Convincing evidence exists that meteoritic matter was reheated shortly after the initial condensation of the solar nebula for those meteorites thought to be derived from parent bodies. This evidence takes the form of cooling rates carefully determined from diffusion studies of the migration rate of Ni across kamacite-taenite boundaries in iron meteorites (Fish, Goles, and Anders, 1960; Goldstein and Ogilvie, 1965; Goldstein and Short, 1967; Wood, 1964). The notion that the irons condensed directly from the solar nebula requires that these measurements and the existence of large Widmanstätten figures be explained as a condensation event. This seems rather unlikely and, in any event, requires a far more complex explanation than heating and melting in a parent body.

The reheating episode and the need for melting at least the outer layers of the Moon early in the evolutionary time track of the solar system led Urey (1961) to propose the existence of a class of radionuclides that are now extinct and whose presence is postulated or inferred from the daughter products that follow naturally from the decay. In the case of ${ }^{26} \mathrm{Al}$, this evidence is difficult to identify because the product of decay is ${ }^{26} \mathrm{Mg}$, which is naturally abundant. Recently, Schramm et al. (1970) determined an upper bound from the examination of feldspars in terrestrial, lunar, and meteoritic samples. They found that the contribution of. ${ }^{26} \mathrm{Al}$ to parent body heating would have been unimportant at the time of solidification. Additionally ${ }^{244} \mathrm{Pu}$ and ${ }^{129} \mathrm{I}$ are known to have existed in the solar nebula because trace quantities of the Xe daughter products have been found and Pu fission tracks have been observed (Fleischer, Price, and Walker, 1968; Reynolds, 1963). Nevertheless, the amounts of these fossil nuclides do not appear to have been enough to yield the requisite level of heating. Such ancillary matters as the storage time in the solar nebula are important. Long before the parent bodies condensed to the point where heat could be stored, there would have been appreciable decay that would not contribute to the heating. An additional criticism of the fossil nuclide hypothesis (in terms of heating) is that if serious mixing of the fossil components in the original nebula took place and if parent bodies were to have 
been melted, then the inner planets would also have experienced this process (Sonett, 1969b). Although the early thermal history of Earth is well masked, the same does not appear to be true of the Moon. If the Moon were formed at a position in the solar system where the mixing requirements were met, then it is difficult to understand why the Moon would not have been completely melted very early. The present evidence does not support such an extreme thermal history but, in fact, suggests that the deep interior was only partially melted at best. The electromagnetic evidence from the Apollo 12 magnetometer suggests a condition in which only the outer several hundred kilometers, corresponding to a mantle, were melted (Dyal et al., 1970; Sonett et al., $1971 c, d)$. Thus unless substantial fractionation of the fossil radionuclide component of the primitive solar nebula took place, the hypothesis of fossil nuclide heating must be regarded as only marginally plausible.

We have examined an alternative hypothesis for early heating of the meteoritic parent bodies. This mechanism is based upon ohmic heating by a primordial sun endowed with high spin, a reasonable magnetic field, and an extreme outflow of plasma that might be loosely characterized as a solar wind. These conditions conform generally to those postulated for $\mathbf{T}$ Tauri stars and also form a self-consistent chronology because $\mathrm{T}$ Tauri stars are thought to be of approximately 1 solar mass in an early postcondensation stage (Sonett, 1969a,b; Sonett, Colburn, and Schwartz, 1968; Sonett et al., 1970).

The process of ohmic heating is based upon the generation of electrical currents deep within the body by the action of the interplanetary magnetic field and the solar wind. Electrical currents flow as a result of excitation from the interplanetary magnetic and electric fields, corresponding to a transverse electric (TE) and transverse magnetic (TM) excitation of the body (Schubert and Schwartz, 1969; Sonett et al., 1971a; Sonett et al., 1971b,c). Detailed calculations have been carried out for the case of steady-state (time-independent) TM excitation. The detailed results of this work have been reported elsewhere and only the final conclusions are given here. These are shown in figure 1 and indicate the peak temperature reached for a representative set of parent body radii, starting temperature $T_{0}$, and solar spin decay "time constant" $\delta$. The latter is incorporated into a subroutine for solar spin damping so that the sun evolves toward the present epoch spin rate while starting at a higher value. Figure 1 shows three basic regimes for the heating process. The left side of the diagram shows the radiation damped cases where losses from the body into space limit the peak temperature. The peak value is achieved in very short times, characteristically some $5 \times 10^{5} \mathrm{yr}$, after which radiative losses dominate and the body cools. The other extreme is given by the case where the heating is joule dominated and radiative losses are important only near the surface. In this range of sizes, the heating varies approximately as $1 / R^{2}$ where $R$ is the body radius. The intermediate cases are governed by more complex phenomena involving lack of saturation. Saturation means that the formation of a bow shock wave ahead of the body is inhibited by the small 


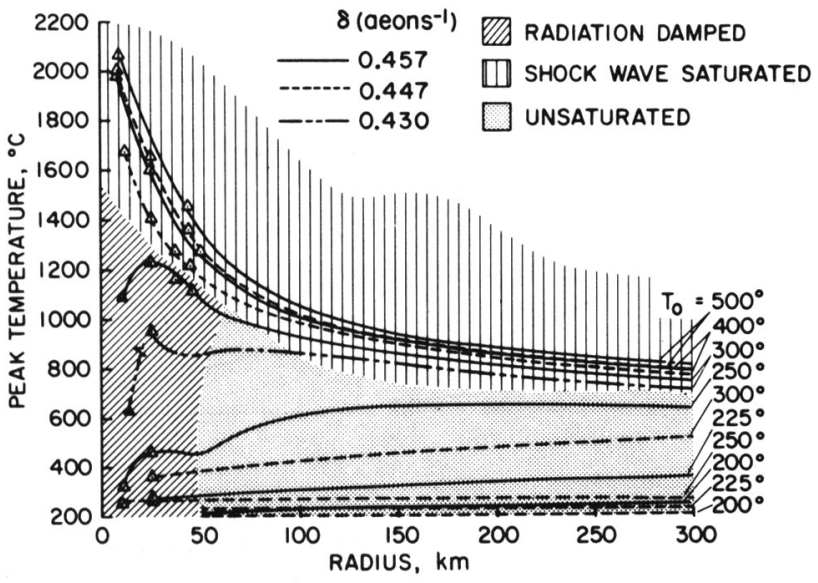

Figure 1.-Peak core temperatures of bodies ranging in size from 10 to $300 \mathrm{~km}$ radius using a bulk electrical conductivity function for basalt-diabase (Parkhomenko, 1967), and for various uniform starting temperatures and a variable solar spin rate. The latter is identified with the solar magnetic field time constant $\delta$ shown above the graph. The largest value corresponds to a starting field of $2.3 \mathrm{mT}(23 \mathrm{G})$ and a centrifugally limited sun; the other values correspond respectively to spin rates of 150 and 80 times the present value. The computer runs carried the computation to $10^{6} \mathrm{yr}$; thus for the smaller bodies that were eventually overwhelmed by radiative losses, the peak temperature is attained early (i.e., about $5 \times 10^{5} \mathrm{yr}$ ) and the triangles correspond to this time rather than $10^{6} \mathrm{yr}$ as in the case of the larger bodies, which are not radiation damped. The heating calculation shown here is for the TM mode and does not include either TE heating or any addition due to fossil nuclides. (Adapted from Sonett et al., 1970).

electrical current. In these cases the $1 / R^{2}$ dependence is voided because it depends exclusively upon the process of saturation where the total planetary current is determined by mechanical pressure effects in turn governed by the radius of the body, other parameters being equal. Details of the heating are shown in figures 2 and 3 . The physical behavior is given in the references.

The calculations reported' here are determined by a wide set of parameters including the surface temperature, which is required because the TM mode currents pass through the surface of the body (Sonett et al., 1970). On the other hand, the TE mode does not carry this restriction. Calculations are underway to determine the effects of coupled TE-TM heating. Generally, it can be said that the addition of the TE mode will increase the heating, although special conditions may prevail during part of the cycle where the two conflict.

A rudimentary example of TE mode heating can serve to illustrate the conditions required for this case. To melt a parent body requires on the order of $10^{4} \mathrm{~J} / \mathrm{g}$ of matter. To do this in about $10^{6} \mathrm{yr}$ (an upper bound for TM heating) would require that the energy input into the body correspond to about $100 \mu \mathrm{W} \cdot \mathrm{m}^{-3}\left(3 \times 10^{-3} \mathrm{ergs} / \mathrm{cm}^{3}-\mathrm{s}\right)$. The target area of a planet is given 


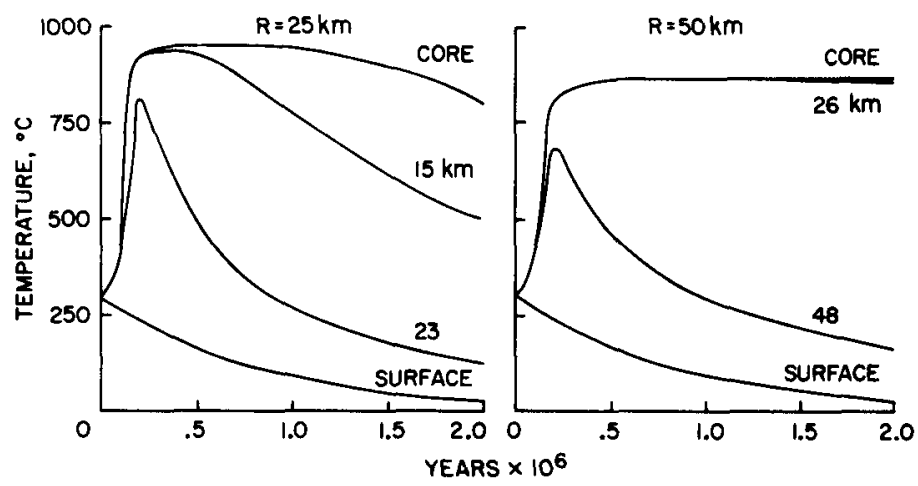

Figure 2.- The detailed chronology of parent bodies having radii $R=25$ and $R=50 \mathrm{~km}$ subject to TM heating alone. The figure shows the temperature versus time at different levels in the bodies measured inward from the surface. It is seen that the surface follows the space temperature closely; at $2 \mathrm{~km}$ depth the temperature rises rapidly at first followed by a strong loss of heat, while the core for $R=25 \mathrm{~km}$ shows only a small decline after 1 million $\mathrm{yr}$ and for $R=50 \mathrm{~km}$ shows no loss in that time. These calculations are for basalt-diabase (Parkhomenko, 1967), $T_{0}=300^{\circ} \mathrm{C}$, and a spin decrement $\delta=0.447$.

by $\pi R^{2}$ whereas the volume is $(4 / 3) \pi R^{3}$; thus the field amplitude requirement is given by the condition that

$$
\frac{B^{2}}{2 \mu} \frac{v}{R}=10^{-4}
$$

where $B$ is the peak value of the field amplitude, $v$ the speed of the solar wind, and $R$ the radius of the body. This equation is a statement of the intercepted flux per unit volume of the body, under the assumption that the incident hydromagnetic radiation is wholly adsorbed through joule heating. Then $B \sim 0.03 \mathrm{mT}(0.3 \mathrm{G})$. This value can be compared to the strength of the basic spiral field for TM heating. For a centrifugally limited sun with a maximum permissible starting magnetic field of $2.3 \mathrm{mT}(23 \mathrm{G})$ yielding the final present epoch spin (assuming an exponential decay in the magnetic field), the field strength at $3 \mathrm{AU}$ at the onset is of an order of $8 \mu \mathrm{T}(80 \mathrm{mG})$. Thus the disturbance field is considerably greater than the steady component, and the interplanetary magnetic field can be characterized as turbulent. This condition is not in conflict with the extrapolation of conditions thought to exist in the emissions from T Tauri stars. The postulated conditions for the steady component of the field can easily be modified upwards because the present model is conservative with respect to the starting magnetic field. Spectroscopic evidence of fields associated with $\mathrm{T}$ Tauri stars is limited by turbulence broadening so that the observational threshold is on the order of $1 \mathrm{dT}\left(10^{3} \mathrm{G}\right)$, 


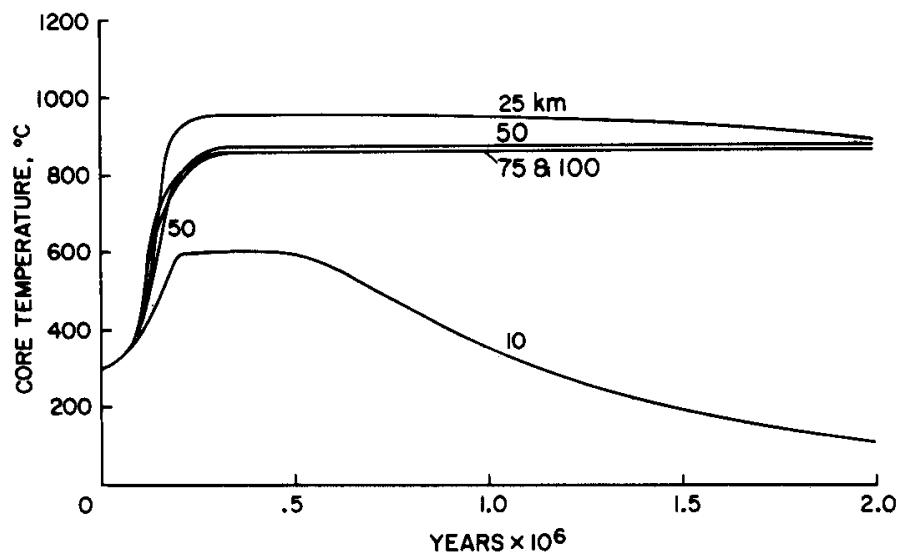

Figure 3. - The core temperature versus time for bodies ranging in size from 10 to $100 \mathrm{~km}$ using TM heating solely. The smallest body $(R=10 \mathrm{~km})$ shows the effect of radiative loss to space most strongly while the larger bodies show little or no effect in 2 million yr. The material assumed, starting temperature, and spin decrement are the same as for figure 2 .

more than two orders above our estimate. Increase of the starting field can substantially increase the interplanetary electric field that drives the TM mode although saturation effects must be considered where the formation of a bow shock wave inhibits further heating. Nevertheless, for those cases where the onset of the bow wave is absent, an additional component of TM heating can be expected; and, in any event, the heating is not expected to be diminished by the saturation process. Then the spiral structure can be preserved even in the presence of an extremum in the time-dependent part of the field, though this is not an essential part of the model.

The conclusions to be drawn from this note are perhaps even more important in regard to the early Sun than for the question of planetary heating. For the absence of an adequate explanation of the $\mathrm{Ni} \cdot \mathrm{Fe}$ phases using radiogenic sources means that the electrical hypothesis becomes more compelling; this in turn requires certain critical conditions to be placed upon the early pre-main sequence Sun. These are essentially the high spin rate, modest to large magnetic field, and the presence of a substantial efflux of plasma, representative of $\mathrm{T}$ Tauri stars. All this is in accord with contemporary models of the early evolution of the solar system where condensations from a primordial cloud accompanied by a Hayashi-like contraction of the Sun are implied.

\section{REFERENCES}

Dyal, P., and Parkin, C. W. 1970, Electrical Conductivity and Temperature of the Lunar Interior From Magnetic Transient Response Measurements. NASA TM X-62012. J. Geophys. Res., in press. 
Fish, R. A., Goles, G. G., and Anders, E. 1960, The Record in the Meteorites. III: On the Development of Meteorites in Asteroidal Bodies. Astrophys. J. 132, 243.

Fleischer, R. L., Price, P. B., and Walker, R. M. 1968, Identification of Pu ${ }^{244}$ Fission Tracks and the Cooling of the Parent Body of the Toluca Meteorite. Geochim. Cosmochim. Acta 32, 21.

Goldstein, J. I., and Ogilvie, R. E. 1965, The Growth of the Widmanstätten Pattern in Metallic Meteorites. Geochim. Cosmochim. Acta 29, 893.

Goldstein, J. I., and Short, J. M. 1967, Cooling Rates of 27 Iron and Stony-Iron Meteorites. Geochim. Cosmochim. Acta 31, 1001.

Parkhomenko, E. I. 1967, Electrical Properties of Rocks. Plenum Press, Inc. New York.

Reynolds, J. A. 1963, Xenology. J. Geophys. Res. 68, 2939.

Schramm, D. N., Tera, F., and Wasserburg, G. J. 1970. The Isotopic Abundance of ${ }^{26} \mathrm{Mg}$ and Limits on ${ }^{26} \mathrm{Al}$ in the Early Solar System. Earth Planet. Sci. Lett. 10, 44.

Schubert, G., and Schwartz, K. 1969, A Theory for the Interpretation of Lunar Surface Magnetometer Data. The Moon 1, 106.

Sonett, C. P. 1969a, Fractionation of Iron: A Cosmogonic Sleuthing Tool, I, Radioisotope Heating. Comments Astrophys. Space Phys. 1, 6.

Sonett, C. P. 1969b, Fractionation of Iron: A Cosmogonic Sleuthing Tool, II, Heating by Electrical Induction. Comments Astrophys. Space Phys. 1, 41.

Sonett, C. P., Colburn, D. S., and Schwartz, K. 1968, Electrical Heating of Meteorite Parent Bodies and Planets by Dynamo Induction From a Pre-Main Sequence T Tauri Solar Wind. Nature 219, 924.

Sonett, C. P., Colburn, D. S., Schwartz, K., and Keil, K. 1970, The Melting of Asteroidal-Sized Bodies by Unipolar Dynamo Induction From a Primordial T Tauri Sun. Astrophys. Space Sci. 7, 446.

Sonett, C. P., Dyal, P., Colburn, D. S., Smith, B. F., Schubert, G., Schwartz, K., Mihalov, J. D., and Parkin, C. W. 1971b, Induced and Permanent Magnetism on the Moon: Structural and Evolutionary Implications. Highlights of Astronomy 1970 (ed., C. De Jager), in press. D. Reidel. Dordrecht.

Sonett, C. P., Dyal, P., Parkin, C. W., Colburn, D. S., Mihalov, J. D., and Smith, B. F. 1971a, Whole Body Response of the Moon to Electromagnetic Induction by the Solar Wind. Science 172, 256.

Sonett, C. P., Schubert, G., Smith, B. F., Schwartz, K., and Colburn, D. S. 1971c, Lunar Electrical Conductivity From Apollo 12 Magnetometer Measurements: Compositional and Thermal Inferences. Proc. Apollo 12 Lunar Sci. Conf. Geochim. Cosmochim. Acta 35, suppl. II:

Sonett, C. P., Smith, B. F., Colburn, D. S., Schubert, G., Schwartz, K., Dyal, P., and Parkin, C. W. 1971d, Lunar Electrical Conductivity Profile. Nature 230, 359.

Urey, H. C. 1961, The Origin of the Moon and Its Relationship to the Origin of the Solar System. Proc. Symp. 14 IAU. Academic Press, Inc. New York.

Wood, J. A. 1964, The Cooling Rates and Parent Planets of Several Iron Meteorites. Icarus 3,429 .

\section{DISCUSSION}

ALFVÉN: It is difficult to see how your mechanism works. You assume that the current is given by the conductivity of the body. Instead, would not the lack of charged particles transferring the current to and from the body be the limiting factor? If so, the energy released by ohmic heating must be small compared to the heating due to direct solar-wind impact on the body.

SINGER: Have you investigated the torque produced in the parent body by the interaction of the induced magnetic moments and the external field?

SONETT: This calculation has not been carried out. It was suggested earlier (Sonett et al., 1970) that fields of the magnitude indicated would have completely despun parent 
bodies. Then if the asteroids are their residues, they should not be spinning today. Repeated collisions, however, could cause spin up where the total ensemble spin angular momentum is zero but both prograde and retrograde spins are present.

SINGER: For one of your modes of heating, it is necessary for currents to flow between the solar wind and the body. Will photoelectrons suffice?

SONETT: It seems most likely that photoelectron emission from the negative hemisphere and positive ion collection on the other hemisphere would suffice for the steady-state TM mode that I discussed. A value of nearly $10^{-3} \mathrm{~A} / \mathrm{m}^{2}$ is given for peak photocurrent (Sonett et al., 1970). The use of the photocurrent mechanism to supply current carriers means, however, that the mechanism is restricted to the sunlit hemisphere, whereas the calculations are for cylindrical symmetry. However, I do not think that the error introduced by the restriction to the sunlit hemisphere is severe.

\section{DISCUSSION REFERENCE}

Sonett, C. P., Colburn, D. S., Schwartz, K., and Keil, K. 1970, The Melting of Asteroidal-Sized Bodies by Unipolar Dynamo Induction From a Primordial T-Tauri Sun. Astrophys. Space Sci. 7, 446. 